\title{
2 Serienkommunikation als Forschungsgegenstand
}

Die Beziehung zwischen medialen Produkten und Kommunikation über mediale Produkte lässt sich mit Keppler (1998: 187) folgendermaßen beschreiben: „Wie die mediale Kommunikation nichts wäre ohne das mediale Produkt, so ist andererseits das Medium nichts ohne seinen sozialen Gebrauch“. Keppler, die komparativ Gespräche über Literatur in alltäglichen Unterhaltungen mit der Fernsehsendung des literarischen Quartetts analysiert, bezeichnet solche Kommunikation als Gespräche über „ästhetische Erzeugnisse“. Ästhetische Erzeugnisse definieren sich ihr zufolge darüber, dass

\footnotetext{
es sich hierbei um Produkte handelt, von denen immer auch so gesprochen wird, daß [sic!] ihre spezifische Machart zur Sprache kommt. (Dies ist natürlich ein sehr weiter Begriff, der von Objekten der Mode über Unterhaltungssendungen des Fernsehens bis hin zu elitären Kunstobjekten reicht; aber einen solchen weiten Begriff braucht es, wenn untersucht werden soll, wie sich etwas als ästhetisches Erzeugnis konstituiert und welcher Stellenwert ihm im individuellen und sozialen Leben zukommt.) (Keppler 1998: 187)
}

In dieser Studie werden Serien als solche zum Gesprächsgegenstand entwickelten, in ihrer medialen Machart wahrgenommenen, ästhetischen Erzeugnisse modelliert. Daher wird in diesem Kapitel zunächst die eine Seite dieses Wechselverhältnisses Serien als spezifische Medienform (Abschnitt 2.1) - beschrieben, bevor in Abschnitt 2.2 der Forschungsstand der anderen Seite - Medienrezeption (Abschnitt 2.2.1) und Kommunikation über Medien (Abschnitt 2.2.2) - dargestellt sowie die vorliegende Studie darin eingeordnet wird (Abschnitt 2.2.3).

\subsection{Serialität und Serienrezeption im Forschungsdiskurs}

Serialität kann als grundlegendes „Wahrnehmungs- und Ordnungsprinzip des Menschen“ (Faulstich 1994: 51) bezeichnet werden. Seit jeher prägen serielle Erzählungen die Produktion und Rezeption von medial-ästhetischen Werken - begonnen bei dem orientalischen Zyklus TAusendundeInE NACHT (Mielke 2006) über Fortsetzungsromane in Zeitschriften wie OLIVER TwIST bis hin zu seriellen und transmedial in Erscheinung tretenden Comic-Figuren wie Batman (vgl. Staiger 2019: 17). So besteht Serialität nach Eco (1999: 301) als „Wiederholungskunst“ stets aus der wiederholten Variation eines Schemas (Eco 1999: 319). Die Faszination für Serien liegt Eco zufolge darin, dass sie die „prophetische Gabe“ ihrer Konsument*innen belohnen: „wir sind glücklich, weil wir unsere Fähigkeit entdecken, das Geschehen 
vorherzusehen“ (Eco 1999: 305). Das derartig in seiner Grundstruktur und -funktion beschriebene serielle Formprinzip bildet mittlerweile den Untersuchungsgegenstand vieler Disziplinen, wie der Literatur- (Giesenfeld 1994) und Medienforschung (Hickethier 1991), der Kultur- (Kelleter 2012) und Kommunikationswissenschaft (Schlütz 2016), der Medienpädagogik (Theunert und Gebel 2000) und jüngst auch der Literatur- und Mediendidaktik (u.a. Anders und Staiger 2016).

Die große Bandbreite serieller Formate versuchen Weber und Junklewitz mit der von ihnen so bezeichneten „Minimaldefinition“ einer Serie zu erfassen: „Eine Serie besteht aus zwei oder mehr Teilen, die durch eine gemeinsame Idee, ein Thema oder ein Konzept zusammengehalten werden und in allen Medien vorkommen können. “ (Weber und Junklewitz 2008: 18). Abseits dieser Definition gibt es viele Bestrebungen, Serialität kriteriengeleitet zu systematisieren, um Serienformate klassifizieren zu können (z.B. Blättler 2003, Schlachter 2016, Schlütz 2016). Dabei handelt es sich um ein Unterfangen, das sich angesichts der Vielzahl an Formaten und der sehr kleinschrittigen Ausdifferenzierung im Produktions-, Präsentations-, Distributions- und Rezeptionskontext oft als schwierig erweist (vgl. auch Staiger 2019: 173).

In den folgenden Abschnitten werden die von den Jugendlichen in der vorliegenden Studie diskursiv verhandelten Aspekte zu Serialität dargestellt. Die Kriterien zur Strukturierung in Form von drei Dimensionen ergeben sich aus theoretischer Perspektive, die folgende Kontexte der Serienrezeption in den Blick nimmt: Erstens narratologische Aspekte (Abschnitt 2.1.1), die sich auf die narrativen Gestaltungsprinzipien und -möglichkeiten gegenwärtiger Serienproduktionen sowie daraus resultierende Konsequenzen für die Serienrezeption durch Individuen beziehen. Zweitens technisch-mediale Aspekte (Abschnitt 2.1.2), die jenseits der Erzählformen serieller Formate beschreiben, wie sich Serienproduktion, -distribution und -präsentation unter den Bedingungen der Digitalisierung verändern und sich auf die Serienrezeption auswirken. Drittens soziokulturelle Aspekte (Abschnitt 2.1.3), mit denen eine gesellschaftliche Perspektive auf Serien eingenommen wird. Denn Serien und Serienrezeption sind nicht nur individuell im Alltag der Rezipient ${ }^{\star}$ innen verortet, sondern auch in größere soziale Strukturen und Wertungen eingebettet.

\subsubsection{Narratologische Aspekte}

Die Charakteristika seriellen Erzählens werden in der Forschungsliteratur vielfältig beschrieben und diskutiert. In diesem Abschnitt werden vorrangig die für diese Studie relevanten Aspekte der Mehrteiligkeit, der Erzähldramaturgie und der Figuren fokussiert. Wie schon aus der oben zitierten Minimaldefinition nach Weber und Junklewitz (2008) hervorgeht (s.o.), ist Mehrteiligkeit einer der konsti- 
tutiven Faktoren für Serialität (vgl. u.a. Hickethier 1991: 8). Die Realisierungsform von Mehrteiligkeit bietet oft einen Ausgangspunkt für Systematisierungsversuche serieller Formate (vgl. z.B. Wolling 2004: 172). In der deutschsprachigen Forschung wird traditionell zwischen Serien, Reihen und Mehrteilern (vgl. Mikos 2014: 4) unterschieden. Die Einstufung einer seriellen Produktion in eines dieser Formate hängt davon ab, inwiefern Handlungen in Episoden abgeschlossen werden und wie sehr sie über ein „gemeinsames Rahmenkonzept“ (Schlütz 2016: 24) verfügen, d.h. inhaltlich und/oder über die Erzählstruktur miteinander verbunden sind.

In der angloamerikanischen Forschung zu TV-Serien wird in Bezug auf die Erzähldramaturgie zwischen „series“ und „serial“ (Kelleter 2012: 25) unterschieden. Serien mit abgeschlossener Episodenhandlung werden als episodic series (Episodenserie) und Serien mit offenen und damit fortlaufenden Handlungssträngen als continuous serial (Fortsetzungsserien) bezeichnet (vgl. u.a. Anders und Staiger 2016: 4, Weber und Junklewitz 2008: 19). Als Beispiel für eine Episodenserie, die kaum fortschreitende narrative Entwicklung aufweist und ihre Episoden primär über die Konstanz des Figurenensembles verknüpft, nennt Schlütz (2016: 19) die Serie The Simpsons. Lost dagegen könnte ihr zufolge als ein Beispiel für eine Fortsetzungsserie gelten, da deren Episoden inhaltlich und über die Erzählstruktur stärker miteinander verbunden sind. Eine weitere Heuristik zur Einordnung von Serienformen schlagen Weber und Junklewitz (2008) vor, indem sie Serien nach ihrer Fortsetzungsreichweite und der Fortsetzungsdichte unterteilen. Die Reichweite gibt an, über welche Distanz sich jeweils ein Handlungsbogen erstreckt, und die Dichte beschreibt das quantitative Verhältnis zwischen den Folgen, die fortgesetzt erzählen und denjenigen, die abgeschlossen sind. (Weber und Junklewitz 2008: 24). Klassifikationen erweisen sich jedoch bisweilen als schwierig, da das „narrative Repertoire des seriellen Erzählens“ - wie Staiger (2019: 173) konstatiert - „ein großes Experimentierfeld für neue Erzählformen“ bietet sowie viele aktuelle Produktionen Hybrid- und Mischformen darstellen. Unabhängig von ihrer Fortsetzungsreichweite und -dichte unterliegen Serien insgesamt dem Paradoxon zwischen Schematisierung, d.h. der Anforderung, eine gewisse Verlässlichkeit für die Rezipient`innen zu bieten, und dem Druck, diese Schemata angemessen zu variieren; kurz, sie müssen „Reproduktion als Innovation [...] betreiben“ (Jahn-Sudmann und Kelleter 2012: 207). Jahn-Sudmann und Kelleter (2012) argumentieren, dass Serienproduktionen zur Lösung dieses Problems Strategien intraserieller und interserieller Überbietungen nutzten. Daran knüpft Schultz-Pernice (2016: 45-46) an und differenziert diese Strategien in Verdeckung, Eskalation, Expansion, Verschiebung und Metaisierung aus.

Narrativ komplexe Serienformate nutzen erzählerische Strategien zur formalen und ästhetischen Gestaltung der Serie, die mit Konventionen und Erwartungen brechen oder neue Erzählformen generieren können (vgl. Mittell 2012: 98). Zentral 
ist für Mittell dabei, dass Rezipient`innen nicht nur von den Inhalten und der Spannung einer erzählten Welt fasziniert sind, sondern auch an komplexeren erzählerischen Verfahren Gefallen und Vergnügen finden. Dazu zählen ihm zufolge zum Beispiel Analepsen und Prolepsen oder das Spielen mit der Unterscheidung von Fiktion und Wirklichkeit. Wenn sich Zuschauer^innen die Frage „Was wird passieren?“"statt „Wie hat er das gemacht?“ stellen, bezeichnet Mittell (2012:111) diesen Rezeptionsmodus als „operationale Ästhetik“. Komplexität gilt in der Serienforschung als ein zentrales Merkmal für so genanntes Quality TV. Thompson verhalf dem Begriff über seine Studie Television's Second Golden Age (1996) zu Popularität, der seither in der Serienforschung vielfach aufgegriffen und diskutiert wurde (u.a. Schlütz 2016, Frizzoni 2012, Jahn-Sudmann und Starre 2013). ${ }^{1}$ Sowohl Mittell (2012) als auch Thompson (1996) beziehen sich vorrangig auf US-amerikanische Produktionen und deren Veränderungen im Zuge der 1990er Jahre. Thompson führt insgesamt zwölf Merkmale von Qualitätsserien an, die Blanchet (2011) ins Deutsche übertragen hat und die hier zusammenfassend aufgezählt werden: Wahrnehmung der Serienmacher als Künstler und der Serie als Kunstform, großes Figurenensemble und multiperspektivisches Erzählen, Kombination konventioneller Genres zu etwas Neuem, literarische Komplexität und Autorenzentriertheit, Selbstreflexivität, Aufgreifen kontroverser Thematiken und Realitätsnähe, Preise, Auszeichnungen und positive Kritiken. Da dieser Katalog Aussagen von Zuschauer`innen, Kritiker`innen und Wissenschaftler ${ }^{\star}$ innen gleichermaßen berücksichtigt, beziehen sich nicht alle Kriterien ausschließlich auf die Erzählweise, sondern beinhalten auch darüber hinausgehende normative Werturteile zur Einschätzung der Qualität von Serien (vgl. dazu auch die Ausführungen in Abschnitt 2.1.3).

Für die Entwicklung serieller Erzählungen werden oft die Figuren als besonders wichtig hervorgehoben. So ist für Weber und Junklewitz (2008: 24) eine gewisse Kontinuität des Figurenensembles ausschlaggebend für die Klassifizierung als Serie und auch Eco (1999: 305) definiert Serialität über eine überschaubare Anzahl an Hauptcharakteren. Mittell (2012: 105) unterscheidet narrativ komplexe Serien danach, dass sich im Unterschied zu Serienformen wie Soaps Beziehungs- und Figurendramen aus dem Plot heraus entwickelten. In diesem Zusammenhang wird auch zwischen runden und flachen Charakteren unterschieden, die sich entweder während einer seriellen Erzählung weiterentwickeln oder überwiegend stereotyp bleiben (vgl. dazu beispielsweise Lecke 2014 oder Denson und Mayer 2012). Hickethier erläutert, dass Figuren in der Regel das Interesse von Rezipient`innen an einer Serie aufrechterhalten, denn „sie spiegeln, machen vor, wie Zuschauer

1 Für eine kritische Perspektivierung auf den Diskurs dazu vgl. u.a. Eichner 2013: 60-61 und für das Verhältnis von Quality TV und Popkultur Hecken und Opp 2017: 166-167. 
sich verhalten können, indem sie sich selbst verhalten“ (Hickethier 2012: 362). Auch Bock (2013: 171) kann im Rahmen ihrer Studie die Identifikation mit Serienfiguren als ein wesentliches Rezeptionsmotiv ermitteln. Ein Grund mag darin liegen, dass die kontinuierliche Fortsetzung von Serien mit gleichbleibenden Figuren ein „emotionales Bezugssystem [ermöglichen kann], das zur Ausprägung von Anhängerschaft und Fansein führt“ (Bendix u. a. 2012: 297). ${ }^{2}$ Einen aufschlussreichen Untersuchungsgegenstand bieten moralisch ambivalente Charaktere wie DEXTER, für den einer Studie von Schlütz et al. (2013) zufolge Rezipient*innen ihre Sympathie mittels diverser argumentativer Strategien rechtfertigen. Insgesamt scheint es aus Publikumsperspektive wichtig zu sein, dass Figuren möglichst vielschichtig und ,echt‘ wirken sollten (vgl. z.B. Bock 2013: 126).

Sowohl die Frage nach der Mehrteiligkeit von Serien als Merkmal der Abgrenzung zu Filmen (Abschnitt 8.1.1) als auch Überbietungsstrategien und deren Auswirkungen auf die Rezeption diskutieren die Proband*innen in den vorliegenden Daten der Studie (vgl. Abschnitt 7.3.2). Ausgefeilte Klassifikationen von Serien spielen für sie zwar eine eher untergeordnete Rolle, allerdings setzen sie doch die Frage nach der Abgeschlossenheit von Episodenserien im Vergleich zu komplexeren Erzählformen (Abschnitt 8.1.3) relevant bzw. diskutieren davon ausgehend über Serialität per se (vgl. dazu auch Weiser-Zurmühlen 2017). Um gleichermaßen sowohl die Teilnehmendenperspektive als auch die graduelle Differenzierung der Erzähldramaturgie zu berücksichtigen, werden in dieser Arbeit die Begriffe episodenorientiert und fortsetzungsorientiert erzählende Serien zur Kategorisierung unterschiedlich komplexer Serien genutzt. Die Bedeutung von Figuren für die Medien- bzw. Serialitätsdidaktik verorten Anders und Staiger (2016: 12-13) auf der Subjektebene des Lesekompetenz-Modells nach Rosebrock und Nix. Damit geht jedoch die Frage einher, wie außerhalb von Fragebogen- bzw. Interviewstudien oder experimentellen Designs ein empirischer Zugriff auf die subjektive Wahrnehmung von Figuren möglich ist. In der vorliegenden Studie wird analysiert, wie die teilnehmenden Jugendlichen Aspekte der Identifikation und Glaubwürdigkeit von Serienfiguren für Positionierungen nutzen (Abschnitt 8.1.4) und in der Interaktion aushandeln. Außerdem wird herausgearbeitet, inwiefern sie die - oft durch Figurenverhalten evozierte - emotionalisierende Wirkung von Serien thematisieren (Abschnitt 8.2.4.1).

2 In der Rezeptionsforschung wird in diesem Zusammenhang häufig das Konzept der Parasozialen Beziehung bzw. Interaktion in den Blick genommen mittlerweile: soziale Parainteraktion (Marx und Schmidt 2018: 17), das auf Horton und Wohl 1956 zurückgeht, vgl.für einen Überblick Hartmann 2010. 


\subsubsection{Technisch-mediale Aspekte}

Das serielle Prinzip prägt seit seinen Anfängen insbesondere das Medium Fernsehen sowie das Verhalten der Zuschauer`innen, deren Rezeption einer Serie sich üblicherweise daran orientiert hat, welchen Sendeplatz die Programmplaner`innen der Serie zugewiesen haben. Umgekehrt passt(e) sich das Medium dem Alltagsrhythmus der Rezipient*innen an (vgl. Mikos 2008: 41). Auch wenn nach wie vor das lineare Fernsehen einen möglichen Distributionsweg für Serien darstellt, geht aus der JIM-Studie 2020 hervor, dass Jugendliche für audiovisuelle - und serielle - Formate am liebsten auf Online-Angebote zurückgreifen. Im Vergleich zu 2019 ist im Zuge der COVID-19-Pandemie im Jahr 2020 die regelmäßige Nutzung (d.h. mindestens mehrmals pro Woche) von Video-Streaming-Diensten von 74\% auf $87 \%$ gestiegen. Netflix und YouTube stehen dabei an erster Stelle (Medienpädagogischer Forschungsverbund Südwest 2018: 45). Vor diesem Hintergrund trifft Giesenfelds Postulat aus dem Jahr 1994, dass die „Serialität der Fernsehserie [...] den Rezeptionsrhythmus vor[schreibt]“ (Giesenfeld 1994: 2), nicht mehr zu. Denn Präsentation, Distribution und Rezeption einer Serie sind nicht mehr so eng miteinander verwoben, dass die Periodizität, mit der Episoden erscheinen, auch zugleich die Zeit ihrer Rezeption bestimmt - ein Faktor, der beispielsweise für serielle Heftromane weiterhin kennzeichnend ist, wie Nast (2017) für die Reihe PERRY RHODAN herausarbeitet. Auch wenn es in den 1990er Jahren bereits die Möglichkeit zur Videoaufzeichnung gab, ergeben sich unter den Bedingungen der Digitalisierung (o.g. Plattformen, Mediatheken, VoD, DVDs und Blu-ray) deutlich mehr Freiheiten, kurz: „Die Rezipierenden sind [...] viel autonomer in ihrer Entscheidung, was sie wann wie oft in welcher Frequenz ansehen“ (Czichon 2019: 20).

Dadurch, dass die Streaming-Anbieter und Mediatheken Serien oft staffelweise zur Verfügung stellen, müssen Rezipient*innen folglich nicht mehr ,auf den Ausstrahlungszeitpunkt einer jeweils neuen Episode [ihrer] Lieblingsserie bis zum nächsten Tag oder sogar eine ganze Woche lang warten“ (Anders und Staiger 2019: 10). Damit geht ein globales Phänomen einher, das als Binge-Watching bekannt ist, in der deutschen Forschungsliteratur häufig mit „Komaglotzen“ (Staiger 2019: 175) oder auch „kompaktem Serienkonsum“ (Schlütz 2016: 134) übersetzt. Es bedeutet dem Online-Wortschatz-Informationssystem Deutsch (OWID) des Instituts für Deutsche Sprache zufolge „das Ansehen mehrerer Episoden einer Fernsehserie (auf DVDs oder per Streaming) hintereinanderweg“. ${ }^{3}$ Eine Einordnung von Binge-

3 Vgl. https://www.owid.de/artikel/407521 [zuletzt abgerufen: 16.03.2021]. 
Watching in den Suchtdiskurs findet sich beispielsweise in einer Einführung zu Medien und Gesundheit von Reifegerste und Baumann (2018: 77):

\begin{abstract}
So kann exzessive Mediennutzung sowohl die körperliche als auch die psychische Gesundheit angreifen. [...] Ähnliche Symptome (Einsamkeit, depressive Verstimmungen, Vernachlässigung sozialer Beziehungen) können auch mit der exzessiven Nutzung von Fernsehinhalten einhergehen. Das Anschauen mehrerer aufeinanderfolgender Episoden von TV-Serien innerhalb kurzer Zeit wird als »Binge-Watching« bezeichnet. Dieser Begriff ist abgeleitet vom »Binge-Eating« oder »Binge-Drinking«, bei dem innerhalb kurzer Zeit größere Nahrungsmengen oder Alkohol aufgenommen werden. [...] Binge-Watching dürfte eher dem Binge-Drinking vergleichbar sein, da es von den betreffenden Personen oft nicht als Problemverhalten anerkannt wird. ${ }^{4}$
\end{abstract}

Mit Czichon (2019: 9) wird im Rahmen dieser Arbeit dagegen von „kumulierter Rezeption" gesprochen, das in wertfreier Art und Weise das Betrachten mehrerer Episoden oder Staffeln am Stück bezeichnet. Da dieses Rezeptionsverhalten gesellschaftlich kritisch gesehen wird (vgl. obiges Zitat), wollten die Autor`innen der JIM-Studie 2018 erstmals von den Jugendlichen wissen, ob sie Serien kumulativ rezipieren würden. Der Studie zufolge machen $65 \%$ der Befragten davon Gebrauch (Medienpädagogischer Forschungsverbund Südwest 2018: 48). Aus didaktischer Perspektive gibt es jedoch Gegenstimmen wie Schlütz (2018), die darauf hinweisen, dass Binge-Watching keine solchen negativen Auswirkungen hat, wie häufig angenommen wird.

Eine weitere Voraussetzung für dieses Phänomen besteht darin, dass Serienrezeption nicht nur zeitlich unabhängig von einer vorgegebenen Programmstruktur geworden, sondern mit der Verbreitung mobiler Endgeräte auch lokal unabhängig geworden ist. Zeigen die Aufzeichnungen der Forscher*innengruppe von Holly et al. (u.a. 2001), ${ }^{5}$ dass soziale Interaktion während des Fernsehens überwiegend im privaten Raum stattfindet - meistens im Wohnzimmer, immer jedoch um den Standort des Fernsehers herum organisiert -, kann dank Laptop, Smartphone und Tablet potentiell überall rezipiert werden. Die Autor*innen der JIM-Studie 2018 interpretieren die „schwindende Bindung an das Fernsehen bzw. Fernsehsender und [die] dort ausgestrahlten Inhalte“ (Medienpädagogischer Forschungsverbund Südwest 2018: 46) daher vor dem Hintergrund des rasant ansteigenden Angebots digitaler sowie online-basierter Nutzungsmöglichkeiten. Da die Distribution serieller Formate dank dieser Entwicklungen nicht mehr auf einen geographischen

4 Für einen Überblick und kritische Diskussion internationaler Studien und journalistischer Texte, in denen das Phänomen problematisiert sowie die Ambivalenz des Diskurses skizziert wird, vgl. Czichon (2019: 2-9).

5 Weitere Informationen zur Studie der Autor^innen werden in Abschnitt 2.2.2 erläutert. 
Raum beschränkt ist, entsteht Eichner, Mikos und Winter (2013: 9) zufolge eine „transnationale Serienkultur“. So sind beispielsweise die Netflix-Produktionen HAUS DES GELDES und DARK nicht nur in Spanien bzw. Deutschland erfolgreich, sondern werden weltweit - wenn auch oft mit Anpassungen für internationale Publika - gestreamt. ${ }^{6}$ Bei vielen Serienproduktionen können zudem sowohl die Audiospuren verändert als auch Untertitel in verschiedenen Sprachen zu- und abgeschaltet werden. ${ }^{7}$

Angesichts der Vielzahl an zur Auswahl stehenden Serientiteln und der Variationsbreite der verschiedenen Plattformen kann nicht nur jede ${ }^{\star}$ r entscheiden, wann und wo sie oder er etwas schauen will, sondern auch was. Seriennutzung stellt also eine Form der aus dem Prozess der digitalen Transformation erwachsenen Individualisierung dar, die mit Bock (2017: 153) als „Form einer nutzerbezogenen Anpassung der Inhalte“ bezeichnet werden kann. Sie erläutert diesbezüglich die kontrastive Entwicklung zum linearen Fernsehen:

Somit finden auch Serienzuschauer_innen mit sehr spezifischen inhaltlichen Präferenzen online Serien, die beispielsweise im Mainstream-TV nur begrenzt angeboten werden, da sie dort als sehr individuelle, auf Nischenpublika zugeschnittene Angebote in der Regel schlechter durch Werbung refinanzierbar sind als die massentauglichen Serienprodukte. (Bock 2017: 153)

Da es sich bei dem in der Studie vorgegebenen Gesprächsgegenstand um keine spezifische Serie handelt, wie beispielsweise die Studie von Bock (2013), die sich auf die Serie CSI - CRIME SCENE InVESTIGATION fokussiert hat, ist dieser Aspekt für das Erkenntnisinteresse der vorliegenden Studie von zentraler Relevanz (vgl. Kapitel 1). Denn wie Gothe und Leichner (2012) mit ihrer Untersuchung zur Interaktion von „Serialisten“ - also „Personen, die sich für Serien ,an sich` bzw. für das Format der Serie begeistern“ (Gothe und Leichner 2012: 173) - in online-Foren zeigen, gehen mit den "Individualisierungs- und Optionalisierungstendenzen“ (Gothe und Leichner 2012: 183) zugleich hochgradig divergente, serienbezogene Wissensstände und Wissensbestände der Interaktionsbeteiligten einher. Die daraus resultierenden interaktiven Konsequenzen und Herausforderungen werden im Rahmen der vorliegenden Studie herausgearbeitet (Kapitel 7). Auch der mit

6 Vgl. Die ZEIT über Netflix in Deutschland (September 2019): https://www.zeit.de/kultur/film/2019-09/tv-streaming-netflix-deutschlandstart-serienbewertung [zuletzt abgerufen: 16.03.2021].

7 So kann beispielsweise mit dem Plugin „Learning Languages with Netflix“ (LLN) das Sprachen-Lernen vereinfacht werden, vgl. Futurezone über Netflix (Februar 2019): https://www.futurezone.de/streaming/article216451099/Du-musst-mehr-Netflixen-wenn-duneue-Sprachen-lernen-willst.html [zuletzt abgerufen: 16.03.2021]. 
der Individualisierung einhergehende Aspekt der Zeit- und Ortsunabhängigkeit spielt für die Studie eine Rolle. So diskutieren die Teilnehmenden die zeitlichen Ressourcen, die sie für Serienrezeption aufwenden (vgl. dazu Abschnitt 8.2.4.3) und sie vergleichen englischsprachige Serien mit deren deutscher Synchronisation (vgl. Abschnitt 8.2.4.4).

\subsubsection{Soziokulturelle Aspekte}

Serienrezeption hängt nicht nur von dem erzählten Stoff des Gegenstands und den digitalen Bedingungen seiner Verbreitung ab, sondern ihr werden auch kulturell geteilte und diskursiv erzeugte soziale Funktionen und Bedeutungen zugeschrieben. Aus der historischen Entwicklung zu Serien als Forschungsgegenstand, wie sie beispielsweise Anders und Staiger (2016) vornehmen, lässt sich schließen, dass Wertungen von Serien als überwiegend populärkulturell ${ }^{8}$ verortete Medienprodukte - und damit auch ihre Rezeption - oftmals auf normativen Urteilen beruhen. So argumentieren die Autor^innen, dass Serien insbesondere in der Germanistik - von wenigen Stimmen abgesehen - ,jahrzehntelang unter einem generellen Trivialitäts- und Manipulationsverdacht“ (Anders und Staiger 2016: 9) standen.

Abgesehen von der divergenten Ausrichtung der Bewertung sind Serien damit durchaus vergleichbar mit gesellschaftlichen und Forschungsperspektiven auf Lesen und Lesesozialisation, bei denen es sich u.a. Groeben (2004: 13) zufolge ebenfalls um einen ,im höchsten Maße wertbesetzten Bereich [...] zwischen Kulturpessimismus und -optimismus“ handelt. Die Kompetenz, Texte verstehend lesen zu können, gilt als wichtige Kulturtechnik und Voraussetzung für gesellschaftliche Teilhabe. Dagegen war die Fähigkeit, alle medialen Erzeugnisse - mit Ausnahme des Films in Form von literarischen Adaptionen - über schriftliche Literatur hinaus rezeptiv und produktiv zu verstehen, lange Zeit dem „Primat der Schriftlichkeit“ (Feilke 2017: 159) untergeordnet und findet erst jüngst explizite curriculare Beachtung. ${ }^{9}$ Wiesner (2014) zeigt eindrücklich auf, dass Jugendliche auf diese Werte- und Normvorstellungen im Hinblick auf Lesen und Schreiben Bezug nehmen, indem sie rekonstruiert, wie diese sich im Rahmen ihrer Interviewstudie dazu positionieren. ${ }^{10}$

8 Zur Einordnung von Serien in den Kontext von Populärkultur vgl. (Hecken und Opp 2017).

9 Vgl. die Strategie der KMK (2018) Bildung in der Digitalen Welt:

https://www.kmk.org/themen/bildung-in-der-digitalen-welt/ [zuletzt abgerufen: 17.03.2021]. 10 Wiesners Konzept der gegenstandsbezogenen Positionierung und dessen Relevanz für die vorliegende Studie wird in Kapitel 3 erläutert. 
Im Hinblick auf Serien dominieren einerseits kulturpessimistische Vorstellungen, wie beispielsweise an der in Abschnitt 2.1.2 dargestellten Wertung der kumulierten Rezeption deutlich wird. Solche Debatten zur ,Mediensucht' sind vergleichbar mit der im 18. Jahrhundert verbreiteten Betrachtung der zunehmenden Lektüre von Romanen in Form eines Krankheitsdiskurses, in dem exzessives Lesen als sog. „Lesesucht“ (vgl. für einen Überblick Wolschner 2012) kategorisiert wurde. Wampfler (2013) zeigt die Parallelen des damaligen und heutigen medialen ,Suchtdiskurses' auf und kritisiert den dafür genutzten Suchtbegriff als undifferenziert und tendenziös, da er sich einerseits überwiegend gegen nicht-privilegierte Gruppen wie Frauen oder Jugendliche richtet und andererseits die dahinterstehende Ideologie durch den digitalen Dualismus - die (physische) Realität und die virtuelle Welt sind als strikt voneinander zu trennende Sphären - gekennzeichnet ist. Andererseits hat sich u.a. mit dem Aufkommen der Cultural Studies und deren kritischer Analysen des Zusammenhangs von Medien, Kultur und gesellschaftlichen Machtstrukturen (vgl. exemplarisch die Beiträge in Hepp und Winter 2012) eine Forschungsrichtung entwickelt, die zum einen mediale Unterhaltungsprodukte von ihrem Trivialitätsverdacht ,freizusprechen' versucht und zum anderen die aktive und partizipative Rolle sowie Gestaltungsoptionen der Rezipient`innen betont, die sich auch in widerständigen ästhetischen Praktiken gegen die als hegemonial behandelte Kultur manifestieren kann (vgl. Storey 2011: 10 und die Ausführungen in Abschnitt 2.2.1). Überspitzt zeigt sich dieser Paradigmenwechsel in dem Titel Everything Bad is Good for you (Johnson 2006), in dem Johnson dafür argumentiert, dass populäre Kultur - insbesondere audiovisuelle Formate - keineswegs ,verdumme“ (Johnson 2006: xi), sondern durchaus herausfordernd für ihre Rezipient`innen sei.

Diese Annahmen spiegeln sich auch in dem in Abschnitt 2.1.1 angeführten Begriff des Quality TV wider. Angefangen bei Thompsons (1996) Merkmalen von Qualitätsserien über das von der DFG geförderte Projekt Ästhetik und Praxis populärer Serialität (2010-2016) bis zu aktuellen Forderungen, eine Serialitätsdidaktik curricular zu verankern (vgl. Staiger 2018, Anders und Staiger 2016, Staiger 2019), zeigt sich, dass die Autor*innen gegen Trivialitätsvorstellungen argumentieren. Allerdings wird dabei oft nur ein bestimmter Typ von Serien ${ }^{11}$ aufgewertet und als Qualitätsserien bezeichnet, dem andere serielle Formen als ,Trash TV‘ gegenübergestellt werden. Exemplarisch zeigen das Frizzonis (2012) Analysen verschiedener, gesellschaftlicher Diskurse zur Bewertung von seriellem Quality und Trash TV. Sie unterscheidet den Spezialdiskurs (Wissenschaft), den Interdiskurs (Medienschaf-

11 i.d.R. US-amerikanische mit hohem Budget produzierte Serien, die sich überwiegend an Erwachsene richten (vgl. Staiger 2019: 174). 
fende) und den Elementardiskurs (Zuschauer*innen), die verschiedene bewertende Perspektiven auf Serien haben. Auch wenn im Spezial- und Interdiskurs Filme und Qualitätsserien höher bewertet werden, spielt diese Differenz im Elementardiskurs keine Rolle - „Serie bleibt Serie“ (Frizzoni 2012: 342). Während also die ästhetischen Qualitäten der neuen TV-Serien im Spezial- und im Interdiskurs rege thematisiert werden, fällt auf, dass sie im Elementardiskurs weit weniger Erwähnung finden. Frizzoni weist auf die Chancen hin, dass Zuschauerinnen und Fans mit der Kanonisierung und den Wertungen von Quality TV aktiv und produktiv umgehen, indem sie sie hinterfragen, modifizieren und parodieren (vgl.Frizzoni 2012: 347-348).

Die Unterscheidung von ästhetisch anspruchsvollen und anspruchslosen Serien beschränkt sich jedoch nicht nur auf die in Abschnitt 2.1.1 dargestellte unterschiedlich komplexe Erzählweise, sondern wirkt sich auch auf Zuschreibungen zu den jeweiligen Rezipierenden aus. So beziehen sich viele Serien(rezeptions)forschende auf die in Ecos Theorien zur Serialität skizzierte Unterscheidung des „naiven“ oder „gewitzten“ Lesers, wobei serielle Medientexte in unterschiedlichem Maße eine „gewitzte Lesart“ bereitstellen (vgl. Eco 1999: 312). Unter anderem basiert darauf Mittells (2012) Konzept der operationalen Ästhetik (vgl. Abschnitt 2.1.1). Auch Schlütz (2016: 173) spricht von „hochwertiger Unterhaltungsrezeption“ beim Konsum von Qualitätsserien, weil sie den Rezipient*innen „mehr als andere mediale Unterhaltungsprodukte [...] kognitive und affektive Herausforderungen bieten“ (Schlütz 2016: 174) würden. Besonders deutlich werden Zuschreibungen an unterschiedliche Rezipient*innen unterschiedlicher Serienformen bei Kumpf (2011: 19), die ,akademische Rezipierende US-amerikanischer Quality TV-Serien in Deutschland“ als „Intellies“ bezeichnet.12 Genau wie sich oben angeführtes DFG-Projekt nicht nur den historischen und narrativen Aspekten von Serialität, sondern auch Fragen der sozialen Distinktion rund um das Thema widmet (vgl. diverse Beiträge in Kelleter 2012), zeigt auch Kumpf (2011, 2013), dass die von ihr Interviewten ihre Serienauswahl und -wahrnehmung zur sozialen Distinktion im Sinne Bourdieus (1982) funktionalisieren. ${ }^{13}$

12 Kumpf selbst definiert den Begriff folgendermaßen: „Der Begriff vereint die Aspekte, intellektuell', wegen des Bildungsgrads der Befragten; ,intelligent', weil den Befragten Serien mit Anspruch wichtig sind; ,telly', kurz für television; sowie ,in-telly', also Fernsehen, das gerade in Mode ist“ (Kumpf 2011: 19).

13 Hier sei allerdings angemerkt, dass Kumpf mit ihrer Probandinnenauswahl und der deduktiv vorgenommenen sozialen Kategorisierung sowie ihrer sprachlichen Bezugnahme auf die jeweiligen Personen - deren Aussagen immer mit ihrem Vornamen, Alter und ihrem Beruf bzw. Studienfach gekoppelt zitiert werden - diesen Befund durchaus selbst mit konstruiert. 
Dem gegenüber steht der Umstand, dass Rezipient*innen Serien zumeist eine hohe individuelle und subjektive Bedeutsamkeit zuweisen. Das liegt einerseits an deren digitalen und ubiquitären Verfügbarkeit und ihrer alltagsstrukturierenden und routinestiftenden Funktion (Abschnitt 2.1.2). Andererseits ermöglichen sie es den Rezipient*innen, durch die über einen längeren Zeitraum hinweg etablierte Nähe zu den Figuren eine persönliche und oftmals langjährige Beziehung zu Serien aufzubauen (Abschnitt 2.1.1). Serien können daher die Funktion von „Alltagsbegleitern, persönlichen Ratgebern und soziokulturellen Orientierungsmarken“ einnehmen (Staiger 2019: 174). Medienpädagogische Studien nehmen bei der Frage nach solchen „soziokulturellen Orientierungsmarken“ häufig genderbezogene Identitätskonstruktionen bei Kindern und Jugendlichen in den Blick (u.a. Pasquier 1996, Illg 2001, Buckingham und Bragg 2005, Franz 2008, vgl. auch den Fokus der meisten Beiträge - neben dem Umgang mit medialer Gewalt - in Theunert und Gebel 2000).

Ein weiterer Aspekt subjektiver Bedeutsamkeit besteht nicht nur in Fragen der sozialen Distinktion, sondern auch in der Herstellung von Gemeinschaft, die über den kommunikativen Austausch über Serien realisiert werden kann (vgl. Binotto und Pfister 2015: 35). In der deutschsprachigen Forschung wird für diese Art von Mediengesprächen oft der Begriff der Anschlusskommunikation (vgl. u.a. Charlton und Sutter 2007) verwendet (vgl. auch Abschnitt 2.2.2). Anders und Staiger (2016: 8) weisen „insbesondere Endlosserien“ vielfältige Möglichkeiten der Anschlusskommunikation zu, die sie „scheinbar unausweichlich auslösen - vom Gespräch auf dem Schulhof bis hin zum Fantalk in sozialen Netzwerken“. Daran wird deutlich, dass Anschlusskommunikation nicht auf face-to-face-Interaktion beschränkt sein muss, sondern auch digital gestützt stattfinden (u.a. Schlachter 2014, Undorf 2012) und damit aktive Partizipation der Rezipient*innen an der Serienproduktion (Kelleter 2012: 15, 28) anstoßen kann.

Die vorliegende Studie setzt an diesem Punkt an und fokussiert solche Anschlusskommunikation unter der Frage nach Prozessen der Vergemeinschaftung und Distinktion. Dabei wird anhand der Daten herausgearbeitet, wie sich die Teilnehmenden sowohl aneinander und ihren jeweiligen Bewertungen von Serien als auch an Aspekten von Serialität an sich orientieren und dadurch dynamische und lokal relevante Identitätsaspekte konstruieren. So bearbeiten die Jugendlichen beispielsweise interaktiv die Phänomene Serien,,sucht“ (Abschnitt 8.2.4.2) sowie (eine zu hohe) emotionale Beteiligung an der Serienwelt (Abschnitt 8.2.4.1). Dabei reproduzieren sie allerdings nicht ausschließlich zugrunde liegende Normen, sondern orientieren sich im Kontext der jeweiligen interaktiven Funktion und Anforderung daran. Um diese Prozesse beschreiben zu können, werden im Rahmen dieser Arbeit Vergemeinschaftung und Distinktion ethnomethodologisch 
konversationsanalytisch perspektiviert und davon ausgehend analysiert, inwiefern sich die Teilnehmenden miteinander über Serien als Gesprächsgegenstand innerhalb der Interaktion vergemeinschaften oder voneinander abgrenzen (Kapitel 7). Allerdings kann diese Ebene nicht losgelöst von dem Phänomen der sozialen Distinktion betrachtet werden, da sich analytisch rekonstruieren lässt, wie die Teilnehmenden auf übergeordnete, diskursive Aspekte (Kapitel 8), wie beispielsweise auf den Trivialitätsdiskurs Bezug nehmen (Abschnitt 8.2.2) - woraus sich wiederum Distinktionsaktivitäten innerhalb der Interaktion ergeben können. Wie mit dem hier beschriebenen Verhältnis zwischen Mikro- und Makroebene in den Analysen umgegangen wird, wird in Abschnitt 4.2 methodologisch reflektiert.

Insgesamt finden die in diesem Kapitel dargestellten narratologischen, technisch-medialen und soziokulturellen Aspekte in den Analysen dann Berücksichtigung, wenn sie von den Teilnehmenden - in konversationsanalytischer Terminologie (vgl. Abschnitt 4.1) - relevant gesetzt werden. Im Folgenden wird zunächst dargestellt, welcher Stellenwert der (Anschluss-)Kommunikation über Medien in der Medienrezeptionsforschung zukommt, bevor speziell gesprächslinguistische Studien zur Kommunikation über verschiedene Medienformen und deren Ergebnisse vorgestellt und diskutiert werden.

\subsection{Kommunikation und Gespräche über Medien im Forschungsdiskurs}

Seit dem Aufkommen der Massenmedien interessieren sich Forschende sowohl dafür, wie diese konsumiert und verarbeitet werden als auch welche Auswirkungen und Effekte sich daraus individuell und gesamtgesellschaftlich ergeben. Um unter dieser Prämisse auszudrücken, dass mit dem Wahrnehmen von Medien ,mehr' als deren reiner Konsum verbunden ist, hat sich - zumindest in der deutschsprachigen Forschungslandschaft - der Begriff der Medienrezeption etabliert. Die Forschungsdisziplinen und -traditionen, die sich der Untersuchung dieses Feldes widmen, sind vielfältig: Von Kommunikationswissenschaft, Psychologie und Soziologie über Literatur-, Film- und Medienwissenschaft zur Medienpädagogik und Linguistik. Deren Verständnis und Konzepte von Medien und deren Rezeption durch Nutzer^innen unterscheiden sich bisweilen erheblich voneinander. Im Folgenden wird zunächst ein Überblick darüber gegeben, wie in der Medienrezeptionsforschung die Rezipient*innen Berücksichtigung finden (Abschnitt 2.2.1), bevor der Fokus verengt wird und solche Studien vorgestellt und diskutiert werden, in denen - überwiegend - aus linguistischer Perspektive kommunizierende Rezipient`innen beforscht werden (Abschnitt 2.2.2). Abschließend werden die Ergebnisse im Hin- 
blick darauf diskutiert, welche Konzepte für die vorliegende Studie in welcher Art und Weise Berücksichtigung finden (Abschnitt 2.2.3).

\subsubsection{Die Rolle der Rezipient*innen in der Medienrezeptionsforschung}

In der Literaturwissenschaft wird unter dem Konzept der Rezeptionsästhetik davon ausgegangen, dass Personen mit einem literarischen Text aktiv interagieren und ihm abhängig von ihrem Textsorten-, Welt- und Vorwissen subjektive Bedeutungen zuweisen (können). Dieser Gedanke prägt auch die Modellierung des Wechselverhältnisses zwischen Massenmedien und ihren Rezipient ${ }^{\star}$ innen. Der damit einhergehende Paradigmenwechsel spiegelt sich in der Umkehrung der Frage „What do the media do with people?“ zu „What do people do with the media?“ (Katz und Foulkes 1962: 378) wider.

Eher quantitativ ausgerichtete, überwiegend in der Kommunikationswissenschaft und Medienpsychologie verortete Ansätze nehmen davon ausgehend die Medienrezipient*innen in den Blick und untersuchen deren „Verarbeitung und Erleben von Medien und medienvermittelten Inhalten“ (Bilandzic, Schramm und Matthes 2015: 11). So interessieren sich die Forschenden beispielsweise dafür, welche medienspezifischen Selektionsentscheidungen unter welchen Voraussetzungen Personen treffen, um bestimmte Bedürfnisse zu erfüllen ${ }^{14}$ oder ihre Stimmung über Medien zu regulieren (im Sinne der Mood-Management-Theorie, vgl. dazu z.B. Zillmann und Bryant 1994). Die in Abschnitt 2.1.1 dargestellten narrativen Gestaltungsmöglichkeiten von Serien - bzw. jeglicher Medienformen - können dazu führen, dass Rezipierende in einen Flow kommen, also gänzlich in die medienvermittelte Geschichte eintauchen (vgl. u.a. Czichon 2019). In diesem Zusammenhang wird auch das Involvement von Rezipierenden untersucht. Damit wird das ,innere Engagement“ aufgrund ,persönlicher Relevanz oder Wichtigkeit der Themen, Objekte oder Ereignisse sowie der in der Botschaft vertretene Standpunkt“ medialer Texte (Donnerstag 1996: 30-31) bezeichnet. Dazu zählen auch Prozesse der Identifikation mit Medienfiguren, das Etablieren einer sozialen Parainteraktion oder -Beziehung mit ihnen (vgl. für einen Überblick über das Phänomen Hartmann 2010). Diese Studien legen nahe, dass Rezipient`innen auch fiktive Erzählungen nach dem von ihnen wahrgenommenen Realismus beurteilen und als mehr oder weniger plausibel einordnen (vgl. z.B. die Studie von Hofer 2016). Studien zur Erforschung dieser Prozesse basieren oft auf experimentellen Settings, Fragebogenstudien, (inhaltsanalytisch ausgewerteten) Interviews oder Gruppendiskussionen. Sie zie-

14 Im Sinne des Use-and-Gratification-Ansatzes, der maßgeblich durch Katz geprägt wurde, von dem auch oben angeführtes Zitat stammt. Zur Kritik daran vgl. z.B. Ayaß 2012: 1-2. 
len eher darauf ab, generalisierende Aussagen über größere (Teil-)Populationen treffen zu können und berücksichtigen selten die konkrete Rezeptionssituation, kontextuelle Faktoren oder Bedingungen sozialer Interaktion. Im Rahmen der vorliegenden Arbeit sind die Ergebnisse insofern relevant, als sich die dort theoretisch bestimmten Konzepte und Begriffe - Selektion von Medieninhalten, emotionales Erleben und Involvement, Identifikation mit Figuren - als von den Interagierenden selbst relevant gesetzte Phänomene herauskristallisiert haben. Die hier vorgenommenen theoretischen Darstellungen dienen so als ,Begriffsinventar‘ zur Analyse, wie die Teilnehmenden diese Aspekte diskutieren (vgl. dazu die Unterkapitel von Abschnitt 8.2).

Für den angelsächsischen Sprachraum leisteten die Cultural Studies (vgl. auch Abschnitt 2.1.3) und insbesondere Stuart Halls (1980) Encoding-Decoding-Modell einen wesentlichen Beitrag dazu, die aktive Konstruktionsleistung der Medienrezipient`innen empirisch zu berücksichtigen. Das Modell verweist auf die ideologiekritische Ausrichtung der Forschungstradition, denn Hall unterscheidet drei medientextbezogene Lesarten von Rezipierenden, die die darin eingeschriebenen (encoded) Bedeutungen dominant (ihrer hegemonialen Bedeutung entsprechend), oppositionell oder ausgehandelt interpretieren (decoding) können. Empirisch umgesetzt wurde dieses Modell von Morley (1980), der im Rahmen einer Gruppendiskussion untersucht, wie sich dominante, oppositionelle und ausgehandelte Lesarten auf die soziale Schicht von Rezipierenden beziehen lassen. Mit Hilfe des Modells wird argumentiert, dass insbesondere Fans populärkultureller Produkte oppositionelle Lesarten zu medialen Objekten kritisch und aktiv entwickeln können und sie so „gegen den Strich“ verstehen (vgl. z.B. Fiske 2011 und Jenkins 1992). Mit den Cultural Studies gehen auch zugleich qualitativ ausgerichtete und stärker den Kontext berücksichtigende Forschungsansätze einher (vgl. z.B. die Studie von Gillespie 1995), um Medienrezeption empirisch zu erfassen. So hat sich auch mit dem qualitativen Forschungsparadigma der Begriff der Aneignung etabliert, ${ }^{15} \mathrm{der}$ versucht, die aktive, individuelle und kontextabhängige Konstruktionsleistung von Medienrezipient*innen - stärker als der Rezeptionsbegriff $-\mathrm{zu}$ betonen und der sich dezidiert gegen Stimulus-Response-Ansätze wendet. Ayaß und Gebhardt (2012: 2) konstatieren dazu:

\footnotetext{
We use the term appropriation to illustrate that media recipients are no empty vessels into which media contents can simply be poured. [...] In the reception situation, media content does just not meet ,users' but everyday actors who first and above all make sense of what they see and hear, and what they read and listen to. Recipients are neither black boxes nor tabulae
}

15 Für eine kritische Diskussion des Konzepts der Aneignung vgl. Geimer 2010: $13-15$ und Geimer 2011. 
rasae. Rather they have their own everyday practices at their disposal, their habits and their stocks of knowledge (in the sense of Alfred Schütz; cf. Schütz und Luckmann 1973). It is against this background that meaning in general is allocated, that media, too, are understood and interpreted. The term appropriation attempts to account for these circumstances.

Aus dem Verständnis von Aneignung als individuelles und vom Kontext abhängiges Phänomen ergeben sich durchaus Schwierigkeiten für deren empirische Erfassung (konkreter: Messbarkeit) in der Kommunikationswissenschaft (vgl. z.B. Gehrau 2002: 44). Auf subjektive Prozesse der Aneignung kann nur durch Introspektion der Individuen und in rekonstruktiver Art und Weise Zugang erhalten werden. Das scheint eine Hürde zu sein, die Forschende in Bezug auf quantitative Studien zu Rezeptionsverhalten und -motivation oft selbst reflektieren. So konstatiert Bock, dass Befragungen eine „Artikulationsfähigkeit und Wissen um den abgefragten Gegenstand voraus[setzen]“, so dass damit „für sich allein“ wenig belastbare Ergebnisse“ generiert werden können, da „artikuliertes Verhalten nicht zwangsläufig tatsächlicher Nutzungspraxis entsprechen“ müsse (Bock 2015: 18). Auch Suckfüll et al. (2002: 196), die mit einer Kombination aus Interview und Fragebogenstudie Rezeptionsmodalitäten zu Filmen herausarbeiten, stellen fest, dass „[d]ie meisten Interviewpartner/innen [...] in der Befragungssituation das erste Mal überhaupt damit konfrontiert [waren]“. Medien werden jedoch nicht nur individuell rezipiert, sondern - wie Kepplinger und Martin (1986) im Rahmen einer teilnehmenden Beobachtung nachweisen konnten -, halten vielfältig Einzug in alltägliche Gespräche. ${ }^{16}$ Da Rezipient*innen im Gespräch einander verbalisieren und interaktiv aushandeln (müssen), wie sie einen bestimmten Medieninhalt verstehen, welche Bedeutung sie ihm zuweisen und wie sie diesen bewerten etc., ergibt sich eine Möglichkeit, über diese Gespräche mit qualitativen Methoden empirischen Zugriff auf kommunikative Aneignungsprozesse (Charlton und Sutter 2007) zu erhalten.

Insgesamt kann mit Bonfadelli (2008: 32) resümiert werden, dass das „Gebiet ,Medienkommunikation und Gespräche“ [sich] als äußerst vielfältig und gleichzeitig stark fragmentiert“ erweist. Er systematisiert Methoden, Forschungsdisziplinen und -ansätze nach den Kriterien Form (mündlich/schriftlich), Anlass (spontanoffen/geplant-strukturiert), Thema (öffentlich-politisch, Medieninhalte, private Themen), Phasen (präkommunikativ, begleitend und postkommunikativ), Modalität (kognitives Verstehen, Interpretation und Sinnkonstruktion, Beeinflussung, Ausdruck/Verarbeitung von Emotionen) und Methodik (quantitativ-standardisiert vs. qualitativ-ethnographisch). Der folgende Forschungsüberblick in Abschnitt

16 Aktuelle Studien zeigen, wie im Zuge der Digitalisierung auch Smartphones als mediale Objekte und Inhalte zugleich interaktiv aufgenommen, eingebettet und verhandelt werden, vgl. z.B. Keppler 2014 oder Suderland 2019. 
2.2.2 widmet sich dieser Heuristik folgend mündlichen, überwiegend offenen Gesprächen, die Interagierende sowohl rezeptionsbegleitend als auch postkommunikativ führen, und die aus qualitativ-ethnographischer Perspektive analysiert werden. Die vorliegende Studie selbst kann in ähnlicher Weise verortet werden, allerdings haben die Gespräche einen etwas weniger offenen Formcharakter und Medien werden darin ausschließlich postkommunikativ thematisiert. Die Analysen zielen dabei dezidiert nicht auf die Rekonstruktion von Aneignungsprozessen ab, sondern nehmen medienbezogene, in ihrem sozialen Kontext situierte Positionierungsaktivitäten auf verschiedenen Ebenen in den Blick.

\subsubsection{Forschungsüberblick: Medienrezeption in der Interaktion}

Diese Arbeit reiht sich in die konversationsanalytische Erforschung von Mediengesprächen ein. Daher wird zunächst ein kurzer Überblick über Forschungsarbeiten zur rezeptionsbegleitenden und -anschließenden Kommunikation aus nichtgesprächsanalytischer Perspektive gegeben, bevor konversationsanalytische Arbeiten von Keppler und Holly et al. zur Fernsehkommunikation, Hausendorf zur Kunstund Ausstellungskommunikation und Gerwinksi et al. zur Theaterkommunikation dargestellt werden.

Häufig beschäftigen sich Studien mit der Kommunikation über mediale bzw. ästhetische Gegenstände, indem sie Personen in Interviews oder Gruppendiskussionen - und ggf. in Kombination mit anderen Forschungsmethoden - zu ihrem ,Anschlusskommunikationsverhalten' befragen. So interessieren beispielsweise Sommer (2010) oder Hefner (2012), wie Nachrichten wahrgenommen und (kommunikativ) verarbeitet werden. Weber (2015) und Geimer (2010, 2011) nehmen insbesondere Jugendliche als Proband*innengruppe in den Blick, um die identitätsstiftenden bzw. sozialisierenden Funktionen der Kommunikation über Medien empirisch herauszuarbeiten. Auch in lese- und literaturdidaktischen Ansätzen (bspw. Philipp 2008, 2011) bildet die Frage nach dem Einfluss von Anschlusskommunikation mit den Peers auf den Erwerb von Lesekompetenz und Lesemotivation das Erkenntnisinteresse.

In diesem Sinne argumentieren auch Charlton und Sutter (2007), die auf der Grundlage schulischer und außerschulischer Gesprächsdaten über epische und lyrische Texte zeigen, dass Schüler ${ }^{\star}$ innen über Strategien der kommunikativen Bearbeitung mehrdeutiger Textausschnitte Bedeutungen und Sinn aushandeln, wechselseitig Verstehen sichern und kreative Lesarten entwickeln. Die Aushandlungsprozesse interpretieren die Autoren auch vor dem Hintergrund der institutionellen Situierung und plädieren für die Notwendigkeit von Anschlusskommunikation im Literaturunterricht. Sie gründen ihre Forschung nicht auf Befragungen, 
sondern auf audiografierte und transkribierte authentische Gesprächsdaten, die sie „gesprächsanalytisch“ (Charlton und Sutter 2007: 14) auswerten. Dabei gehen sie allerdings nicht nach den Prinzipien der ethnomethodologischen Konversationsanalyse oder Gesprächsanalyse vor (vgl. zur methodischen Vorgehensweise im Rahmen dieser Arbeit Abschnitt 4.1). Im Gegensatz dazu arbeiten die Autor`innen der Beiträge in dem Sammelband von Ayaß und Gerhardt (2012) ethnomethodologisch und rekonstruieren die - alltägliche - Aneignung von Medien in rezeptionsbegleitender Interaktion, beispielsweise zu Videospielen (Piirainen-Marsh 2012, Mondada 2012), Castingshows (Spreckels 2012) oder Fußballspielen (Gerhardt 2012).

Als maßgebliche Studie, die Medienrezeptionsforschung und Gesprächslinguistik verbindet, ist Über Fernsehen sprechen: Die kommunikative Aneignung von Fernsehen in alltäglichen Kontexten von Püschel, Holly, Bergmann et al. zu nennen. Im Rahmen des gleichnamigen DFG-Projektes (1995-1997) zu fernsehbegleitender Interaktion widmeten sich die Forscher*innen nicht der Frage, wie Personen nach der Medienrezeption über Medien kommunizieren - also im Wortsinn der Anschlusskommunikation, die sie als sekundäre Thematisierung bezeichnen -, sondern währenddessen (bezeichnet als primäre Thematisierung; vgl. Hepp 1997: 192-193). Unter der Hypothese, dass Fernsehinhalte kommunikativ angeeignet werden, indem Rezipierende währenddessen in vielfältiger Weise über Sendungen sprechen, prägten die Autor`innen insbesondere für den deutschsprachigen Forschungsraum den Begriff der Aneignung für medienspezifische Interaktion (vgl. Ayaß 2012: 2). Das Studiendesign beinhaltet sowohl Audioaufnahmen von Fernsehgesprächen aus insgesamt vierzehn Tagen in sieben unterschiedlich großen Zuschauergruppen, die nach Alter, regionaler Herkunft und sozialem Milieu unterschiedlich zusammengesetzt wurden, als auch die Videomitschnitte der Fernsehsendungen. Das viele Stunden umfassende Material an „Rezeptionskommunikation“ (Klemm 2004: 192) wurde gesprächsanalytisch nach ihren Formen und Funktionen ausgewertet. Die Studie wurde breit rezipiert ${ }^{17}$ und für aktuelle Forschungen fruchtbar gemacht. So zeigen beispielsweise Androutsopoulos \& Weidenhöffer (2015) eindrücklich, wie sich die damaligen Erkenntnisse auf digitale Kontexte übertragen lassen: Sie untersuchen rezeptionsbegleitende Tätigkeiten des Twitterns zum TATORT und kommen zu dem Schluss, dass sich ähnliche Muster finden, die sie mit medienspezifischen Kategorien ergänzen. Dabei verstehen sie insbesondere Bewerten - bei Klemm eine Handlungskategorie unter mehreren -

17 Beispielsweise von Breidenstein (2006: 111-118), der überzeugend dafür argumentiert, dass es nicht zu übersehende Parallelen zwischen dem Publikum vor dem Fernseher und Schüler^innen vor der Lehrkraft im Frontalunterricht der Schule gibt. 
als übergeordnete Kategorie, da „potentiell jeder Twitter-Beitrag zum laufenden Spielfilm u.E. als explizit oder implizit bewertend gelesen werden kann“ (Androutsopoulos und Weidenhöffer 2015: 34).

Als weiteres umfangreiches Projekt ist die Studie von Gerwinski, Habscheid, Linz et al. zur Publikumskommunikation im Theater (vgl. v.a. Beiträge in Gerwinski, Habscheid und Linz 2018) zu nennen. Die Forschenden analysieren gesprächsanalytisch-rekonstruktiv kommunikative Praktiken der Zuschauer^innen in Pausen- und Foyergesprächen auf der Grundlage eines Datenkorpus mit Audioaufnahmen mehrerer Pausen- und Foyergespräche in zwei Theatern. Unter der Prämisse, dass die publikumsseitige Aneignung der performativen Darbietung in dem Sinne funktioniert, dass das Theater zur „,Reflexion über die eigene Lebenswelt und die gesellschaftliche Wirklichkeit“ werden kann (Habscheid und Linz 2018: 6), modellieren sie Kommunikation im Theaterfoyer als an der Schnittstelle zwischen öffentlicher Kunstinstitution und Small Talk (vgl. Habscheid 2018). Sie zeigen, dass im Unterschied zur medienbegleitenden Kommunikation retrospektive, rekonstruktive und verbal-sprachliche Verfahren auf die ästhetische Darbietung verweisen (vgl. Schlinkmann 2018) und dass sich die Präferenzstrukturen für Bewertungen von ihrer interaktiven Organisation in alltäglichen Gesprächen unterscheiden (können) (vgl. Hrncal 2018).

Keppler (1994) analysiert Gespräche über Medien - überwiegend Fernsehinhalte, aber auch Musikstücke und Literatur - im Rahmen ihrer Studie zu Familieninteraktionen. Die zwei von ihr erforschten Familien wurden gebeten, selbst das Audioaufnahmegerät zu bedienen, wenn sie gemeinsam Mahlzeiten einnehmen. Die so audiografierten Tischgespräche untersucht sie aus der Perspektive der Analyse Kommunikativer Gattungen (Günthner und Knoblauch 1997) und geht u.a. der Frage nach, wie Medieninhalte zu Gesprächsthemen werden und welche Bedeutung ihnen für die Familieninteraktion zukommt (Keppler 1994: 212). Sie plädiert dafür, Kommunikation über Fernsehinhalte nicht losgelöst von dem sie umgebenden sequenziellen Gesprächskontext zu analysieren, denn „das kommunikative Potential der Medien kann nur erfassen [...], wer in der Lage ist, die alltägliche Kommunikation über Medien zu erfassen“ (Keppler 1994: 32-33).

Keppler unterscheidet zwischen ,eingebauten“ Medienreferenzen auf der einen und eigenständigen Medienrekonstruktionen auf der anderen Seite, ${ }^{18}$ die sich vor allem auf gesprächsstruktureller Ebene unterscheiden. Eingebaute Medienreferenzen in Form von Kurzverweisen und Belehrungen kennzeichnen sich strukturell dadurch, dass sie dem eigentlichen Gesprächsthema untergeordnet

18 Für eine anschauliche tabellarische Übersicht von Medienverweisen und Medienrekonstruktionen nach Keppler und Ulmer/Bergmann vgl. Schlinkmann 2018: 313. 
sind. Mit Kurzverweisen ziehen die Interagierenden medial vermitteltes Weltwissen hinzu, um in persuasiver und/oder argumentativer Art und Weise Phänomene der Alltagswirklichkeit zu erklären. Sie können dadurch auch Themen verlagern oder Konflikte bearbeiten. Belehrungen dienen konkret dazu, eine Wissensasymmetrie zu medial vermittelten Wirklichkeitsbereichen zu bearbeiten. Ihre sprachlichinteraktive Struktur ist Keppler zufolge ähnlich zu Belehrungen in anderen kommunikativen Kontexten; der Unterschied besteht primär in den Medien als Herkunft der Wissensquelle. $\mathrm{Zu}$ den so „eingebauten“ Medienreferenzen resümiert sie: „Der bloße Rekurs auf Medienbeiträge vergrößert die Reichweite der familiären Themen, ohne die Struktur der Gespräche zu verändern“ (Keppler 1994: 234).

Bei Medienrekonstruktionen stellen dagegen massenmediale Ereignisse ein eigenständiges Gesprächsthema dar. Keppler unterteilt die Rekonstruktionen in Re-Inszenierung, Mehrstimmige Rekonstruktion, Medien der Aktualisierung und Gemeinsame Interpretation. Eine Re-Inszenierung bedeutet, dass einzelne Episoden des Medientextes in Form von wörtlichen und prosodisch markierten Zitaten nachgespielt und bewertend und/oder interpretierend kommentiert werden. Mehrstimmige Rekonstruktionen sind daran erkennbar, dass sie zumeist eine explizite Eröffnungsphase haben und dazu genutzt werden, um vorhandenes Wissen und Interesse zu klären. Re-Inszenierungen weist Keppler eine kommemorative Funktion $\mathrm{zu}$ - Ziel ist also die Pflege gemeinsamer Erinnerungen und Herstellen von Vergnügen -, während Mehrstimmigen Rekonstruktionen ihr zufolge eine akkumulierende Funktion zukommt, d.h. zusätzlich zur Unterhaltung und zum Vergnügen dazu dient, Informationen zu generieren.

Medien entfalten dann eine weniger erinnernde und stärker bedeutungsgenerierende Funktion, wenn sie entweder dazu genutzt werden, gemeinsame oder kontroverse Ansichten oder Einstellungen zu aktualisieren oder gemeinsam die medial vermittelten Inhalte zu interpretieren und Deutungen sowie Bewertungen auszuhandeln. Den Unterschied zu Erzählungen von familiär geteilten Ereignissen und Erlebnissen verortet sie in der kommunikativen Struktur: Es müssen sowohl ein angemessener Kontext hergestellt und das Ereignis schrittweise rekonstruiert als auch explizite Deutungen des medialen Ereignisses ausgehandelt werden. Keppler kommt zu dem Fazit, dass die Funktion von Medien für Tischgespräche zwischen kommemorativer Reinszenierung, akkumulierender Vergegenwärtigung und interpretierender Aneignung variiert. Sie argumentiert dafür, gleichermaßen den Gesprächskontext sowie die Medienästhetik als Einflussfaktoren auf Kommunikation über Medien zu berücksichtigen (vgl. Keppler 1994: 259). 
Der Aspekt der Ästhetik findet besonders bei Hausendorf (et al.) in den linguistischen Analysen zum Sprechen und Schreiben über Kunst Berücksichtigung. ${ }^{19}$ Hausendorf versteht Kunstkommunikation als musterhaft strukturierte Kommunikation, die sich mit konversationsanalytischen Mitteln in ihren Formen und Funktionen beschreiben lässt, aus denen sich wiederum verschiedene soziale Positionen im Kunstbetrieb ergeben. In Anlehnung an seine mit Quasthoff vorgenommene Untersuchung zur Modellierung des Erwerbs von Erzählkompetenz (Hausendorf und Quasthoff 2005; vgl. Hausendorf 2011: 524) analysiert er Kommunikation über Kunst als bestehend aus kommunikativen Aufgaben (vergleichbar mit den „Erzähl-Jobs“), Mittel und Formen. Mit „kommunikativen Aufgaben“ sind keine von außen vorgegebenen Instruktionen gemeint, sondern im konversationsanalytischen Sinne werden „Erscheinungsformen der Kommunikation über Kunst als Antworten auf zugrunde liegende Fragen [verstanden]“, die man „rekonstruierend aus den Antworten zu entdecken hat“ (Hausendorf 2012: 99).

Die Antworten (und Fragen), die Hausendorf im Kontext von Kunstkommunikation rekonstruiert, sind: Bezugnehmen (Worum geht es?), Beschreiben (Was gibt es zu sehen (hören, tasten, schmecken, riechen)?), Deuten (Was steckt dahinter?), Erläutern (Was weiß man darüber?) und Bewerten (Was ist davon zu halten?). Diese Aufgaben werden mit pragmatisch-semantischen Mitteln gelöst, die sich wiederum in vielfältigen lexikalischen und grammatikalischen Formen manifestieren können (siehe dazu die im Vergleich zu älteren Darstellungen modifizierten Abbildungen in Hausendorf (2011: 524) oder für eine ganzseitige Ansicht Hausendorf (2012: 101)) Sie beziehen sich gleichermaßen auf mündliche und schriftliche Kommunikation über Kunst.

Hausendorf zeigt daher nicht nur, wie die Besucher^innen von Ausstellungen interaktiv gemeinsam diese Aufgaben, vor dem Kunstwerk ${ }^{6}$ bewältigen, ${ }^{20}$ sondern auch, dass sich diese Aufgaben allen Akteur ${ }^{\star}$ innen im Kunstbetrieb stellen. Dazu zählen die Künstler^innen selbst sowie die weiteren an einer Ausstellung beteiligten Personen, aber auch professionelle Kunstkritiker`innen oder mit Kunst arbeitende Pädagog*innen oder Therapeut*innen. Er argumentiert, dass es sich dabei um soziale Rollen handelt, die - mehr oder weniger flexibel - soziale Positionen im Kunstbetrieb einnehmen können. Hausendorf (2012: 100) begründet diese Begriffswahl folgendermaßen:

19 Vgl. Hausendorf 2007 und generell Beiträge in Hausendorf 2007, v.a. Kindt 2007, und ThimMabrey 2007, auch: Hausendorf 2012, Hausendorf 2011, Hausendorf und Müller 2015 sowie Beiträge in Hausendorf und Müller 2016.

20 Seine Analysen beziehen sich u.a. auf Besucher*innen der Ausstellung Jugo Exhibition One in Zürich aus dem Jahr 2007, deren Daten er gemeinsam mit Studierenden im Rahmen eines Seminars „Kunstgespräche“ videografiert, transkribiert und ausgewertet hat (vgl. Hausendorf 2007: 26-27). 
Wir ziehen es vor, an dieser Stelle von sozialen Positionen zu sprechen und wollen damit hervorheben, dass sich Sprechweisen zugunsten erwartbarer kommunikativer Strukturen verfestigen und verselbständigen, auf die man im Sinne einer charakteristischen Position referieren kann. Musterhaft ausgeprägte Sprechweisen machen es möglich, dass Sprecher und Sprecherinnen positionsbezogen zugeordnet, dass positionsspezifisch Eigenschaften und Verhaltensweisen zugeschrieben und dass Personen positionsspezifisch bewertet werden können.

Kunstbezogene soziale Positionen differenziert er aus in die des Kunstbetrachters, -kenners, -kritikers, -sammlers und -richters. Indem Personen die beschriebenen Aufgaben mit unterschiedlichen Mitteln und Formen bearbeiten, wirken sie zugleich (auch) an der Konstituierung entsprechender Positionen mit (vgl. dazu die tabellarische Übersicht in Hausendorf 2012: 120f.), denn „kommunikative Muster [sind] immer auch Indikator und Faktor sozialer Positionierungen der Kunstkommunikation“ (Hausendorf und Müller 2016: 9). So können beispielsweise die Ausstellungsbesucher*innen durch die Wahl der sprachlichen Formulierungen als Kunstbetrachtende fremdpositioniert werden (vgl. Hausendorf und Müller 2015: 439-442). Insgesamt verortet Hausendorf die Besonderheiten des Sprechens über Kunst in dem Gegenstand selbst, der spezifische kommunikative Aufgaben erfordert und zur Konstitution sozialer Positionen beiträgt.

\subsubsection{Funktionen und Bedingungen des Kommunizierens über Medien}

Aus den referierten Studien lässt sich ableiten, dass die Analyse von Kommunikation über Medien bestimmte Bedingungen und Spezifika dieser Interaktionsform berücksichtigen muss. Das betrifft sowohl Medien als Gegenstand selbst als auch die Art und Weise, wie über Medien gesprochen wird, und welche Funktionen damit erfüllt werden. Diese drei Aspekte werden im Folgenden zunächst literaturgestützt skizziert, um sie danach jeweils in Bezug zu der vorliegenden Studie zu setzen.

So fungieren Medien für alltägliche Gespräche oft als thematische Ressourcen, über die gemeinsames Vergnügen bzw. Geselligkeit hergestellt werden kann (z.B. Keppler 1994: 234, Klemm 2000: 209-210). Über Medienthemen können Interagierende unverbindlichen Small Talk abhalten (vgl. zur Besonderheit von Small Talk im Theater Habscheid 2018) und somit auch dann ins Gespräch kommen, wenn sie einander nicht gut kennen (vgl. Zitat von Binotto und Pfister 2015 in der Einleitung) bzw. nur eine kurze gemeinsame Interaktionshistorie haben. Des Weiteren kommt Medien auch eine gesprächsorganisierende Funktion zu, denn, wie Ulmer und Bergmann (1993: 89) anhand von Gesprächsausschnitten zeigen, 
greifen Sprechende „,vorzugsweise dann [auf Medien zurück], wenn das Gespräch zu verebben droht oder bereits zum Erliegen gekommen ist.“

Im Kontext von Gesprächen über (massen-)mediale Produkte beziehen die Forschenden nicht nur die sprachlichen Handlungsmuster, kommunikativen Praktiken, Interaktionsstrategien usw. ein, sondern sie berücksichtigen auch den $\mathrm{Ge}$ genstand selbst als „ästhetisches Erzeugnis“ (Keppler 1998187; vgl. auch Kapitel 1) So greifen die Autor ${ }^{\star}$ innen für die Untersuchungen der Foyergespräche auf Erkenntnisse der Theaterwissenschaft (vgl. Linz 2018: 203, Habscheid und Linz 2018: 5) zurück, zur Analyse der Gespräche über mehrdeutige Texte werden Grundlagen aus der Literaturwissenschaft und der Lese- und Mediensozialisationsforschung hinzugezogen (Charlton und Sutter 2007: 45-48) und die Kunstkommunikation kommt als interdisziplinäres Forschungsfeld nicht ohne ihren Bezug zur Kunstwissenschaft aus (u.a. Hausendorf 2007: 20-21, Hausendorf 2011: 512). Auch die Analysen fernsehbegleitenden Sprechens legen nahe, dass sich die Sprachhandlungen je nach Genre der Sendung unterscheiden (können) (Klemm 2000: 345-346). Für die Analyse der Interaktion zwischen Rezipient^innen medialer Produkte erscheint diese Schnittstelle besonders wichtig. So argumentiert Hepp (1997: 207), dass Zuschauer*innen beim Sprechen über mediale (bei ihm: fernsehbezogene) Inhalte vielfältige „Bezüge zwischen den ,globalen“ Mediendiskursen und der eigenen Lebenswelt her[stellen]“. Er weist den Cultural Studies folglich zu, dass sie die „herausragende Rolle“ interpersonaler Kommunikation bei der Rezeption (und Aneignung) medialer Inhalte verkennen würden (Hepp 1997: 195). Umgekehrt sollten konversationsanalytische Studien nicht die medialen und ästhetischen Besonderheiten des Gesprächsgegenstandes ignorieren (vgl. dazu Keppler 1994: 184). Diese Schnittstelle bezeichnet Keppler (1994: 263-267) als „Relais der Medien“, die zwischen privaten und öffentlichen Orientierungen vermitteln können. Sie gilt es bei der empirischen Erfassung von Medienrezeption in der Interaktion zu berücksichtigen. Inwiefern dieser Zusammenhang in der vorliegenden Studie modelliert wird, wird u.a. in Abschnitt 6.5 dargestellt.

Keppler argumentiert, dass für Familien, die sich auf Themen der kulturellen Öffentlichkeit beziehen, Medien eine wichtige Funktion für die Vergemeinschaftung einer Familie übernehmen, indem sie deren Informationsstand, Ansichten und Einstellungen zu erweitern vermögen. Zentral für das Verständnis von familialer Vergemeinschaftung ist ihr zufolge jedoch nicht, dass die Familie in allen Bewertungen und Einschätzungen medialer und medial vermittelter Inhalte übereinstimmt, sondern dass vielmehr ein „Konsens des Verfahrens“ herrscht, d.h. dass sich alle Beteiligten darüber einig sind, wie sie mediale Themen und Ereignisse kommunikativ behandeln. Zugleich kann genau darüber auch eine Abgrenzung der Familienmitglieder untereinander hergestellt werden (Keppler 1994: 181). Dieses Wechselspiel zwischen Gemeinschaft und Abgrenzung klingt auch in anderen 
Untersuchungen an (vgl. z.B. Baldauf und Klemm 1997: 16). Auch Charlton \& Sutter (2007: 137) subsumieren in ihren Analysen unter der Überschrift ,,andere kommunikative Ziele“, dass die Schüler^innen beim Diskutieren von Gedichten nicht nur Textverstehen und Deuten anstreben, sondern auch Gemeinschaft über scherzhaft gerahmte Interaktion etablieren. Zudem können sich ebenfalls „Koalitionen“ innerhalb der Gruppe - in dem von ihnen analysierten Beispiel zwischen Jungen und Mädchen - ergeben (vgl. Charlton und Sutter 2007: 138-139).

In Gesprächen über Theatervorstellungen lässt sich ein schmaler Grat zwischen wechselseitiger Bestätigung von höflicher Gemeinschaft einerseits und sozialer Distinktion andererseits feststellen. Habscheid (2018: 183) stellt in diesem Zusammenhang die Relevanz des Aspekts der Partizipation heraus:

Die Theoriebildung, die wir uns bisher angeschaut haben, betonte im Blick auf Small Talk und Konversation die verbindenden, inklusiven Wirkungen - Stichworte waren das Herstellen von Gemeinschaft, eine freundliche und harmonische Atmosphäre, Respekt und Anerkennung, das höfliche Vermeiden von Gesichtsbedrohungen, Geselligkeit. Wenn es um konkrete gesellschaftliche Verhältnisse geht, gehört zum Gesamtbild allerdings auch, dass Small Talk und, viel mehr noch, Konversation auch einen exklusiven, ausschließenden Charakter haben können. Der Grund hierfür ist, salopp gesagt, dass nur derjenige mitmachen kann, der weiß, wie es geht. Anders gesagt: die Partizipation an einer Praxis der Vergemeinschaftung setzt entsprechende Skills voraus.

Aus diesem Zitat geht hervor, dass das Herstellen von Gemeinschaft oder Abgrenzung und Partizipation an der Gesprächssituation - die über die reine Ko-Präsenz Interagierender hinausgeht - eng zusammengehören. Beide Phänomene lassen sich mit Hausendorfs (2012) Ansatz verbinden, soziale Distinktion über Positionierungen zu untersuchen. Denn Rezeptionskommunikation dient Charlton und Klemm (1998: 10-11) zufolge „,dem Positionieren in der Welt. Sie sorgt für die Vergewisserung und den Abgleich von Einstellungen, Werten und Wissen, letztlich damit für die Vergemeinschaftung bzw. Differenzierung in der jeweiligen Gruppe.“ In dieser Arbeit wird Positionierung als analytisches Werkzeug verstanden, um Prozesse der Vergemeinschaftung und Partizipation zu untersuchen (vgl. zum Konzept der Positionierung Kapitel 3). Insgesamt lassen sich dem in den Studien jeweils angedeuteten Komplex um Positionierungen und Partizipation zwei wesentliche Aspekte entnehmen: Erstens das Wissen sowohl über Erfordernisse und Art der Kommunikation als auch über den Gegenstand an sich. Zweitens das Mitteilen und Abgleichen von Einstellungen und Werten sowohl bezüglich des Gegenstandes als auch der damit verbundenen Diskurse, Einstellungen und Werturteile - somit kommt also auch dem Bewerten eine tragende Rolle zu. Beide Punkte werden im Folgenden näher erläutert. 
Die Relevanz des Bewertens zeigt sich unter anderem in der Weiterentwicklung der Sprachhandlungskategorien von Klemm bei Androutsopoulos und Weidenhöffer (2015: 34), die die Ubiquität von Bewertungen betonen. Auch Hausendorf hebt die herausragende Stellung des Bewertens hervor. So sei „das bewertende (Geschmacks- oder Rang-)Urteil der eigentliche Fluchtpunkt der Kunstkommunikation“ (Hausendorf 2007: 35, vgl. auch für eine ähnliche Formulierung Hausendorf 2012: 95). Kunstbezogenes Bewerten kann sich ihm zufolge auf verschiedene Weisen manifestieren. Als Antwort auf die der kommunikativen Aufgabe zugrunde liegende Frage „was davon zu halten ist“ kann es sich auf die subjektiv empfundene Wirkung oder den Eindruck des Werks beziehen, die Beurteilung seiner ästhetischen oder handwerklichen Qualität oder auch dessen relationale rang- oder wertbezogene Einordnung im Verhältnis zu anderen Werken oder Künstlerinnen (vgl. Hausendorf 2012: 115). Im Kontext von Theaterkommunikation können die Autor^innen unter Verweis auf Hausendorf ebenfalls zeigen, „dass das Bewerten den Foyergesprächen im Theater von den Gesprächsteilnehmerinnen und Gesprächsteilnehmern als zentrale Aufgabe zugeschrieben und diese Aufgabe von ihnen als geteiltes Wissen vorausgesetzt wird.“ (Linz 2018: 536).

So analysiert Hrncal (2018) detailliert, wie die Zuschauer*innen in den Pausengesprächen die Theaterstücke interaktiv bewerten. Dabei zeigt sich nicht nur die schon erwähnte Umkehrung der Präferenzstruktur von Bewertungssequenzen, sondern auch, dass die Interagierenden ihre Bewertungen abschwächen und viele Heckenausdrücke, Subjektivierungen und Gradationsmarker verwenden. Zudem machen sie selten Gebrauch von tiefergehenden Ausführungen oder Begründungen bzw. Explikationen ihrer Bewertungen. Nach Kindt (2007: 57) liegt der unterschiedliche Begründungszwang von Bewertungen an verschiedenen Erwartungen, die an die Kunst gerichtet sind und die von Vorerfahrungen und persönlichen Interessen abhängen. Ihm zufolge spielt es für die Explikation einer Bewertung eine Rolle, ob Interagierende eine „rationale Diskussion über Kunst“ oder „emotionale Exaltation“ anstreben (vgl. Kindt 2016: 214). Hrncal (2018: 542-543) argumentiert in diesem Zuge über die spezifische Beschaffenheit des Gegenstands der Konversation. Denn wenn ästhetische Kategorien Ziel von Qualitätsurteilen werden, können sie auch in Form von subjektiven Empfindungen und Wertungen heraus artikuliert werden; theoretisches Wissen ist dafür nicht zwingend notwendig.

Damit wird der zweite Aspekt angedeutet, nämlich die Frage, welches und wie viel Wissen für die Partizipation an Rezeptionskommunikation hinreichend und notwendig ist. Zunächst kann konstatiert werden, dass in dieser Hinsicht ein Unterschied zwischen primärer oder sekundärer Medienthematisierung besteht. So können beim gemeinsamen und gleichzeitigen Betrachten eines Films, eines Gedichts oder eines Kunstwerks alle Beteiligten - theoretisch - voneinander annehmen, dass sie darüber in gleichem Maße Bescheid wissen. Allerdings 
muss nun wieder berücksichtigt werden, dass es sich um ästhetische Erzeugnisse handelt, die in besonderem Maße ein individuelles Verständnis, verschiedene Deutungsmöglichkeiten und subjektive Interpretationen zulassen (dazu: Charlton und Sutter 2007: 75-117), die nicht als geteiltes Wissen angenommen werden können. So erläutert Schlinkmann (2018: 369), dass Interaktionsbeteiligte bei sekundären Thematisierungen von Theatervorstellungen permanent kommunikativ abgleichen, „was sie wahrgenommen haben und inwieweit ihre Wahrnehmungen kongruent sind“. Zudem handelt es sich bei Literatur, Kunst, Theaterstücken usw. um Gegenstände, mit denen sich Personen auch in professioneller Hinsicht auseinandersetzen und als Expert*innen bzw. Lai`innen über graduell mehr oder weniger theoretisches oder Hintergrundwissen verfügen können. Diesem Umstand trägt Hausendorf (2012) mit den von ihm beschriebenen sozialen Positionen Rechnung, die er nach dem zugeschriebenen epistemischen Status sortiert - von Kunstbetrachtenden über -kennende $\mathrm{zu}$-kritiker*innen.

Im Zuge der Wissenskommunikation medialer Inhalte greift oft der interaktive Mechanismus des Rekonstruierens. ${ }^{21}$ Aus den diesbezüglichen Analysen von Keppler (1994) und Schlinkmann (2018) lässt sich zusammenfassend festhalten, dass Medienrekonstruktionen einerseits evaluativ oder deutend ausgerichtet sein können und damit wie oben beschrieben Vergnügen, Geselligkeit, der Kommemorierung und Vergemeinschaftung dienen können. Das ist häufig der Fall, wenn die Interagierenden einen ähnlichen epistemischen Status haben, also über das gleiche Wissen verfügen sowie dieses auch wechselseitig voneinander annehmen. Andererseits kann Rekonstruktionen aber auch die Funktion zukommen, Wissensasymmetrien zu bearbeiten, also einander zu informieren oder zu belehren (vgl. auch weitere Ausführungen dazu in Abschnitt 4.3). Im Zuge von Theaterkommunikation können sich nach Schlinkmann (2018) informierende Rekonstruktionen entweder auf Deutungen des Stücks, Verständnisfragen zur Aufführung oder die Vermittlung von Hintergrundwissen beziehen. Dabei kann zwar nicht uneingeschränkt davon ausgegangen werden, dass der rekonstruierte Inhalt allen Beteiligten bekannt ist, allerdings würde es auch zu kurz greifen, in diesem Zusammenhang lediglich von wissenden und nicht-wissenden Personen auszugehen. So zeigt Keppler (1994: 241) anhand des WATZMANNS, dass unterschieden werden kann zwischen „Primär-Wissenden“, Personen, die über Medienwissen aus zweiter Hand verfügen und daher nicht gänzlich unwissend sind, und solchen, die den rekonstruierten Inhalt überhaupt nicht kennen. Diejenigen, die Wissen vermitteln, nehmen eine epistemisch höhere Position ein und bearbeiten - mit Hausendorf gesprochen -

21 Nach Ulmer und Bergmann (1993: 100) handelt es sich dabei um keine eigenständige kommunikative Gattung, d.h. die im Titel aufgeworfene Frage wird verneint. 
die kommunikativen Aufgaben der Rezeptionskommunikation auf eine andere Art und Weise. Dabei müssen Interagierende nicht notwendigerweise einen hohen epistemischen Status als präferiert behandeln. Vielmehr können Personen auch „naiv“ rezipieren, also das ästhetische Werk (Ausstellungsstück, literarischer Text, Film, Theateraufführung etc.) auf sich wirken und sich davon unterhalten lassen, kurz: sie können sich auch als „ein bisschen dumm“ (Ullrich 2007: 213) positionieren.

Dennoch lässt sich erkennen, dass es Situationen gibt, in denen eine Präferenz für mehr Wissen zu erkennen ist - nämlich dann, wenn es um das Verhältnis zwischen Bewerten und Wissen geht. Denn Qualitätsurteile von Medien können Keppler (1994: 239) zufolge unter anderem die Funktion von „Kleinst-Belehrungen“ übernehmen. Hrncal (2018: 264-265, 273) arbeitet heraus, dass über die Organisation der Bewertungen in den Pausenkommunikationen das „epistemische Recht zur Evaluation“ beansprucht werden kann. Dabei wird interaktiv ausgehandelt, wer den höchsten epistemischen Status innehat und somit Expertise für die Abgabe eines bewertenden Qualitätsurteils eher beanspruchen kann und wem diese Expertise interaktiv auch zugeschrieben wird. Dieses Verhältnis zwischen Bewerten und Wissen in der Interaktion wird in Abschnitt 4.3 theoretisch ausführlicher dargestellt, während in Kapitel 7 die diesbezüglichen Analyseergebnisse nachzulesen sind.

\subsection{Zusammenfassung: Serienkommunikation als Forschungsgegenstand im Rahmen dieser Arbeit}

Insgesamt ergeben sich folgende Konsequenzen für die Studie: Der Gesprächsgegenstand in Form von Serien, Serialität und Serienrezeption (Abschnitt 2.1) mit ihren technisch-medialen, narratologischen und soziokulturellen Aspekten findet in der Analyse Berücksichtigung als ,Begriffsinventar‘, um Positionierungspraktiken angemessen beschreiben, einordnen und interpretieren zu können. Die untersuchte Kommunikationsform wird vor dem in Abschnitt 2.2 skizzierten Forschungsstand reflektiert als Gespräche, die postkommunikative, sekundäre Medienthematisierungen beinhalten. Da es sich nicht um Alltagsgespräche, sondern um eine für Forschungszwecke arrangierte und videografierte Interaktionssituation handelt, werden diese Aspekte methodologisch reflektiert (vgl. Abschnitt 5.3).

Aus der Analyse der vorliegenden Daten nach den Prinzipien der ethnomethodologischen Konversationsanalyse (Abschnitt 4.1) lässt sich ableiten, dass die Teilnehmenden Partizipation (Abschnitt 6.2.1) an den Gesprächen anstreben und sich nuanciert miteinander innerhalb der Gruppe vergemeinschaften oder vonein- 
ander abgrenzen (Abschnitt 6.2.2). Positionierung wird hier also weniger als das Einnehmen sozialer Positionen mit definierten ,Rollen' (im Sinne der Studien von Hausendorf als Kritiker, Sammler usw.) betrachtet, sondern als ein dynamischer Prozess, der sich lokal auf die Gesprächssituation bezieht (Kapitel 3 und 6). Von Hausendorfs Typologie wird jedoch der Grundgedanke übernommen, dass interaktive Positionen sich je nach angezeigtem und zugeschriebenem epistemischen Status unterscheiden können. Daher gilt das Erkenntnisinteresse der Analysen der Ausdifferenzierung dessen, wie die Jugendlichen sich epistemisch positionieren, um Bewertungen vorzunehmen, sowie den damit einhergehenden gesprächsstrukturellen Auswirkungen. Denn um an Serienkommunikation partizipieren zu können, ist es notwendig, Bewertungen und Wissenskommunikation als kommu-nikative Aufgaben zu bearbeiten (Abschnitt 4.3 und Abschnitt 6.3). 